Final Report

U.S. Department of Energy

\title{
Real-Time Broad Spectrum Characterization of Hazardous Mixed Waste by Membrane Introduction Mass Spectrometry
}

\author{
Principal Investigator: Charles W. Wilkerson, Jr. \\ Institution: Los Alamos National Laboratory \\ Collaborators(Institutions):
}

Todd M. Allen, Philip H. Hemberger, Mary E. Cisper (Los Alamos National Laboratory);

Richard B. Timmons (University of Texas - Arlington);

Anthony J. Borgerding (University of North Dakota)

Project Number: 59981

Grant Project Officer: Dr. Roland F. Hirsch

Project Duration: 1 October 1997 - 20 September 2000 


\section{Contents}

1 Executive Summary $\quad 3$

2 Research Objectives 3

3 Methods and Results 4

3.1 General Experimental Features . . . . . . . . . . . . . . . . 4

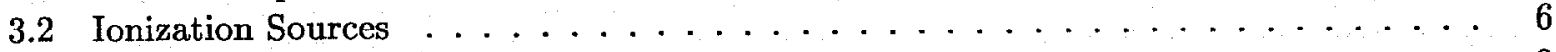

3.3 Simultaneous Detection $\ldots \ldots \ldots \ldots \ldots \ldots$

3.4 Tailored Membranes . . . . . . . . . . . . . . . . . . . 12

4 Relevance, Impact and Technology Transfer 17

5 Project Productivity $\quad 20$

6 Publications $\quad 20$

7 Interactions $\quad 20$

8 Transitions $\quad 21$

9 Patents 21

10 Future Work $\quad$ 21

11 References $\quad 21$ 


\section{Executive Summary}

The goal of this project was to expand the range of chemical species that may be detected by membrane introduction mass spectrometry (MIMS) in environmental, and specifically in Mixed Waste, monitoring and characterization applications. There were three major thrusts to this work:

1. Investigation into improved ion sources for increased analyte sensitivity.

2. Development of methods for simultaneous detection of volatile organics (VOCs), semivolatile organics (SVOCs), and organometallic species.

3. Application of tailored membrane chemistry for compound specificity.

While the application of plasma source ionization was not successful, charge exchange chemical ionization was very effective due to the ability to use abundant matrix species $\left(\mathrm{O}_{2}\right.$ or $\left.\mathrm{H}_{2} \mathrm{O}\right)$ as reagent ions. We have made the first simultaneous measurement of VOCs, SVOCs, and organometallic compounds in air and water matrices. Further, our work on the development of chemically tailored membrane coatings has the potential to facilitate the deployment of a highly selective sensor for hazardous constituents in Mixed Wastes.

This work has resulted in 5 peer-reviewed publications, 5 presentations at national meetings, and 3 invited talks at universities. This research is poised for further development and deployment, but such activities are not currently funded.

\section{Research Objectives}

The goal of this project was to expand the range of chemical species that may be detected by membrane introduction mass spectrometry (MIMS) in environmental, and specifically in Mixed Waste, monitoring and characterization applications. Membrane introduction mass spectrometry (MIMS) [1] functions as a near real-time monitor: there is little to no sample preparation and analysis time is seconds to minutes. MIMS can be implemented as a flow injection technique, where samples, standards, and method blanks can be sequentially analyzed in a continuous fashion. The membrane acts as an interface between the sample (air or water) and the vacuum of the mass spectrometer. Transport of the analyte through the membrane occurs by the process of pervaporation. $[1,2]$ This process is described by adsorption to the outer surface of the membrane, diffusion through the membrane, and desorption from the inner membrane surface into a helium gas flow or into vacuum.

The driving force for this work is the need for a rapid, sensitive, and broadly applicable tool for characterizing organic and metal-containing contaminants in a variety of DOE (and other) waste streams. In all characterization scenarios, a balance must be struck between evaluation of the hazards and their cxtent at a waste site, and the resources available for the overall mitigation of that risk. In the case of chemically, physically, and geometrically homogeneous waste, the situation is aided by the ability to reasonably assume that any sample collected is representative of the overall site constituents. However, few real environmental challenges are homogeneous. As a result, detailed sampling plans must be prepared, and chemical analyses must be performed on a number of samples in order to identify areas of contamination and assess further options. For many years, the chemical analysis part of this process has been accomplished by delivering the samples to a (typically) physically remote laboratory, where very detailed, and concomitantly expensive (both in time and money), procedures have been applied to the samples to determine their content; Environmental Protection Agency (EPA) methods for analyzing wastes for hazardous chemicals are 
time-tested and very reliable. However, in many cases both time and resources may be conserved by being able to make survey analyses at the waste site to determine if any or all samples need to be exhaustively characterized by laboratory-based EPA analytical methods.

A variety of groups worldwide are working on MIMS as a rapid screening tool for a variety of applications; please see our review paper [1] for a detailed overview of these efforts.

Our primary innovations, developed during this project, have been the optimization of the ion source for environmental analyses and the study of chemically tailored pervaporation membranes for enhanced selectivity and sensitivity.

\section{Methods and Results}

There were three major thrusts to this work:

1. Investigation into improved ion sources for increased analyte sensitivity.

2. Development of methods for simultaneous detection of VOCs, SVOCs, and organometallic species.

3. Application of tailored membrane chemistry for compound specificity.

Each of these areas will be discussed below, after a brief introduction to common experimental methods.

\subsection{General Experimental Features}

Figure 1 shows the basic experimental schematic diagram for MIMS. A closeup of the membrane

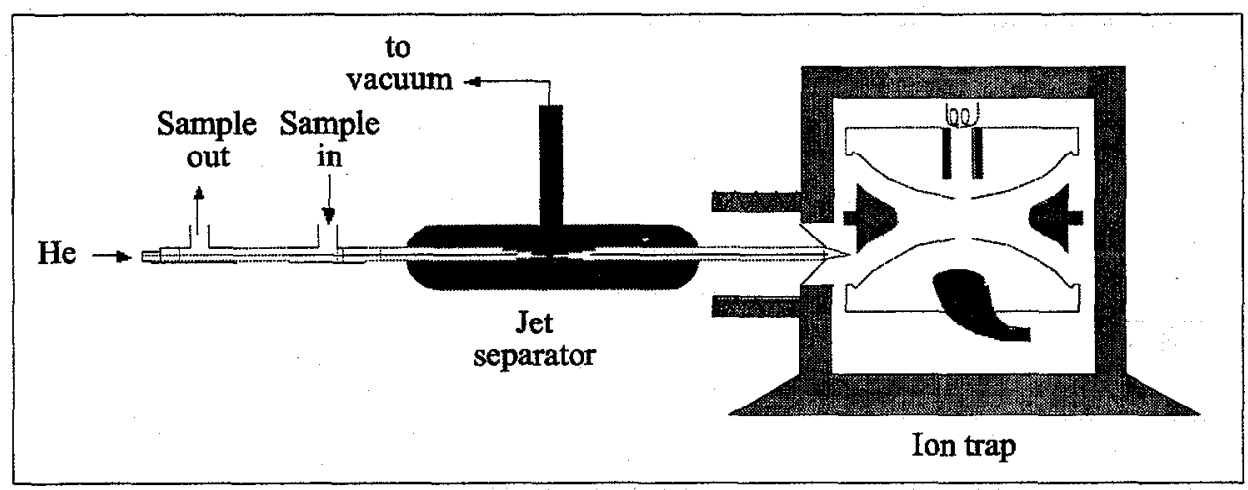

Figure 1: Schematic Representation of MIMS Apparatus

assembly is shown in Figure 2. The membrane assembly is constructed from simple and inexpensive materials. Using vacuum epoxy (Varian TorrSeal), two parallel strands of the membrane fiber were sealed within two Swagelok tees joined by a $5 \mathrm{~cm}$ length of $1 / 4$ inch o.d. Silcosteel tubing. Experimentally, we have found that two strands of the fiber provide an acceptable compromise between analyte signal and excessive matrix pervaporation. The length of each fiber is approximately 10 $\mathrm{cm}$. All of the membrane fibers are based on a hollow microporous polypropylene capillary $(209 \mu \mathrm{m}$ i.d. $x 263 \mu \mathrm{m}$ o.d.). Depending on the experiment, the fiber coating is either polydimethylsiloxane (PDMS, obtained commercially from NeoMecs Inc., Eden Prairie, MN) or a custom coating prepared in the laboratory of Professor Richard B. Timmons, University of Texas - Arlington. All of 


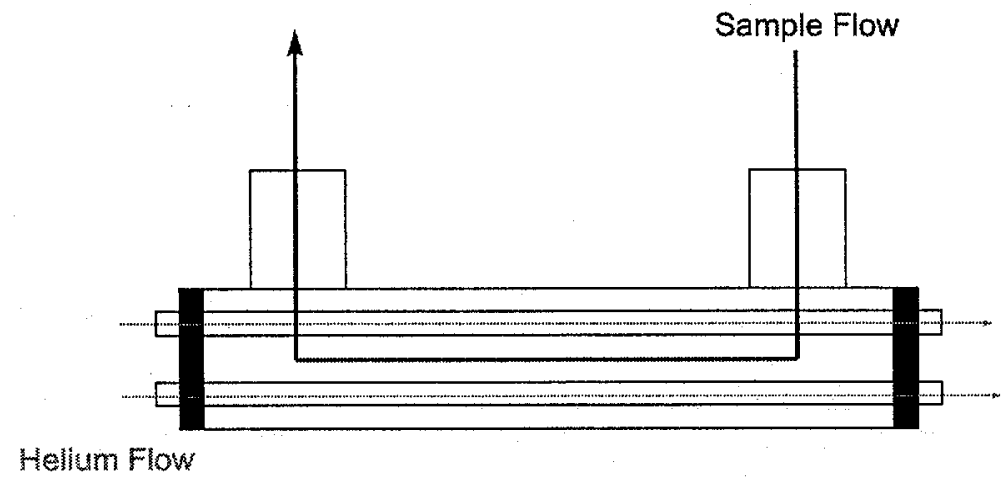

Figure 2: Detail of membrane assembly.

the coatings are applied to the support fiber via plasma polymerization. In the case of the commercial PDMS membranes, the polymer coating is $0.5 \mu \mathrm{m}$ thick. The custom fibcrs have varying thickness, but are believed to be on the order of a few $\mu \mathrm{m}$. The gross (macroscopic) surface area for the membrane fibers is $\sim 0.8 \mathrm{~cm}^{2}$.

Ultrapure helium, regulated by a variable leak valve (Granville-Phillips, Model 203, Boulder, $\mathrm{CO}$ ), flows through the interior of the fibers and serves as both sample transport gas and ion trap buffer gas. A significant increase in instrument performance is realized when He is present in an ion trap mass spectrometer to allow cooling of translational degrees of freedom in the analyte ions.[3]

Cooks and co-workers [2,4] first implemented the use of a metal jet separator for sample enrichment following membrane introduction. In our experiments, a metal jet separator $(P / N$ 113617, SGE, Inc., Austin, TX) heated to $100^{\circ} \mathrm{C}$ connects the downstream side of the membrane assembly to the ion trap manifold, which was held at room temperature.

Aqueous solutions and air samples were detected in a modified Finnigan Model 800 Ion Trap Mass Spectrometer (San Jose, CA) using either charge exchange ionization, electron impact ionization, or plasma (microwave discharge) ionization. The scan function in an ion trap mass spectrometer is designed to create, store, and detect ions through a programmed sequence of events. The scan function requires user tuning of these individual steps via the instrument's operating software to optimize analyte detection and to minimize space-charge effects or unwanted ion/molecule reactions resulting from membrane-diffused analyte, water, and/or air. Membrane-diffused water [5] and oxygen [6] have been used as charge exchange (CE) reagent ions for detecting VOCs, SVOCs and organometallic compounds in air or water (vide infra). A supplemental waveform (nominally $530 \mathrm{kHz}, 6 \mathrm{~V}$ peak-to-peak from a Hewlett-Packard 3312A function generator) was applied to one endcap to implement resonant ejection. Ion trap manifold pressure was typically $2-3 \times 10^{-5}$ torr.

Stock solutions of analytes were prepared using HPLC grade solvents. Trace concentration sample solutions were prepared by serial dilution. Samples were passed over the membrane at a flow rate of (typically) $5 \mathrm{~mL} / \mathrm{min}$ using a peristaltic pump (Model 7524-10, Cole-Parmer Instrument Co., Niles, IL). HPLC grade water was used for establishing the background signal between sampling periods for aqueous samples. Air samples were prepared using a VICI Metronics Dynacalibrator (Model 340-24-Y, Santa Clara, CA). Room air, which was passed through an internal charcoal filter, was used to supply the Dynacalibrator. Analyte concentrations in air were calculated based on chamber temperature, dilution flow rate $(50$ to $450 \mathrm{~mL} / \mathrm{min})$ through the permeation chamber, and diffusion vial capillary length and opening. Air flow over the membrane was regulated to match the flow of the Dynacalibrator by means of a small diaphragm pump at the sample outlet of the 
membrane assembly. The response time lag of the membrane to methanol was about 30 seconds when the air flow rate was $200 \mathrm{~mL} / \mathrm{min}$ (air samples travel $\approx 3$ meters from Dynacalibrator to the membrane assembly). Excess sample flow from the Dynacalibrator and sample effuent were vented through charcoal filters.

\subsection{Ionization Sources}

There are many techniques for generating gas phase ions for analysis by mass spectrometry. In this project, we have looked at three different ionization strategies. A summary of each of these methods is given in Table 1.

\begin{tabular}{|l|l|l|}
\hline Source & Fragmentation & Reaction \\
\hline Electron Impact (EI) & High & $M+e^{-} \longrightarrow M^{+}+2 e^{-}$ \\
\hline Chemical Ionization (CI) & Low & \\
- Charge Exchange (CE) & & $X^{+}+M \longrightarrow X+M^{+}$ \\
- Protonation & & $M+A H^{+} \longrightarrow M H^{+}+A$ \\
\hline Microwave Plasma (MIP) & Variable & \\
- Thermal Ionization & & $M+e^{-} \longrightarrow M^{+}+2 e^{-}$ \\
- Penning Ionization & & $A r^{*}+M \longrightarrow A r+M^{+}+e^{-}$ \\
- Charge Exchange & & $A r^{+}+M \longrightarrow A r+M^{+}$ \\
\hline
\end{tabular}

Table 1: Overview and salient features of ionization methods studied.

Electron impact ionization (EI), is the most commonly used technique in mass spectrometry. Electrons are produced by emission from a hot filament, followed by acceleration to a desired kinetic energy. They are then focused into the ionization region of the spectrometer. Becausc of the high energy interaction involved, EI often results in the deposition of significant internal energy into the analyte ion, which subsequently leads to fragmentation of the ion into a distribution of thermodynamically stable products. Chemically similar species often produce (qualitatively) the same fragments, making data analysis more difficult, particularly for MIMS where there is no significant separation of analytes (i.e. the mass spectrometer must be used for both identification and quantification).

Chemical ionization (CI) uses EI to produce "reagent ions" which then react with neutral analyte species to produce the desired "product ions." Since the reagent ion is usually a molecular species, is typically thermodynamically stable, and has significantly lower kinetic energy than the electron that generated it, CI is often called a "soft" ionization method, because very little fragmentation is seen. This is desirable for MIMS because the molecular ion can be used to identify the species in the sample. A special type of CI, charge exchange ionization (CE, see Table 1) is particularly useful for our work, since we can take advantage of constituents in the matrix (i.e. air and/or water) as reagent ions. While the polymer membrane acts as a semi-permeable barrier between a sample and the vacuum of the mass spectrometer, the overwhelming prevalence of oxygen and water in air and liquid matrices, respectively, leads inevitably to the permeation of these species through the membrane. We have turned this potential liability into a significant advantage by exploiting the ability of the ion trap mass spectrometer to perform controlled gas phase chemistry. The water or oxygen is used to generate chemical reagent ions that enhance analyte signals anywhere from 2 to 20 times over those obtained from more traditional electron impact ionization, with a concomitant reduction in detection limits. In the water $\mathrm{CE}$ process, $\mathrm{H}_{3} \mathrm{O}^{+}$reagent ions are formed through electron impact ionization of membrane-diffused water, water vapor in air samples, or residual 
water in the ion trap mass spectrometer. The $\mathrm{H}_{3} \mathrm{O}^{+}$ions are then allowed to react with the analyte to form abundant $[\mathrm{M}+\mathrm{H}]^{+}$ions. Charge exchange ionization in conjunction with MIMS provides a sensitive method for detecting compounds in air and/or water, and is simple to implement.[7]

In the second year of the project, significant effort was directed towards the development of a microwave induccd plasma (MIP) ionization source interfaced with MIMS. The motivation for this work was the fact that such discharge ionization techniques are often very efficient (up to $5 \%$ ), [8] which would further reduce the method detection limit. In addition, we originally believed that success with standard ionization techniques might be thwarted by two potential problems. The first was the concern that oxygen diffusing through the membrane (particularly in air samples), would result in high partial pressure of oxygen within the ion trap and rapid destruction $(\approx 2$ weeks) of the ionizing filament required in either the electron ionization or chemical ionization modes. Such a short filament lifetime would be unacceptable for an on-line monitoring technique. The second potential issue was associated with the development of on-line chemical derivatization methods for metal containing analytes. It is likely that solvent and/or derivatizing agents would be efficiently transported through the membrane, and the introduction of these compounds to the mass spectrometer could lead to interferences from solvent-derived reagent ions. Spectral interferences from this source would depend on both the solvent and/or derivatizing system and the target analytes.

Therefore, as an alternative to the conventional EI or CI techniques we attempted to employ a microwave discharge for ionization. Membrane permeate is transferred through the jet separator to the discharge region by passing support gas across the membrane surface (pneumatic transport), a concept introduced by Budde and Slivon[9]. Solvent-dominatcd ion source chemistry (as is seen in EI/CI) was expected to give way to chemistry based on the discharge gas. This ionization source is coupled to the ion trap via a linear radio-frequency quadrupole (see Figure 3). By adjusting the $\mathrm{DC}$ and RF potentials on the linear quadrupole, we can select ions of a particular mass-to-charge ratio $(\mathrm{m} / \mathrm{z})$ prior to their introduction to the ion trap.[10] In this fashion, we can reduce space charge effects, which should lead to improved mass spectral quality in terms of peak shape and mass measurement accuracy,[11] and mass selective storage should provide lower detection limits by allowing the selective accumulation of only those ions of analytical interest.[12]

A picture of the quadrupole lens/ion trap combination from the microwave plasma ion source system is shown in Figure 3. The overall length of the instrument in the photograph is $\sim 35 \mathrm{~cm}$.

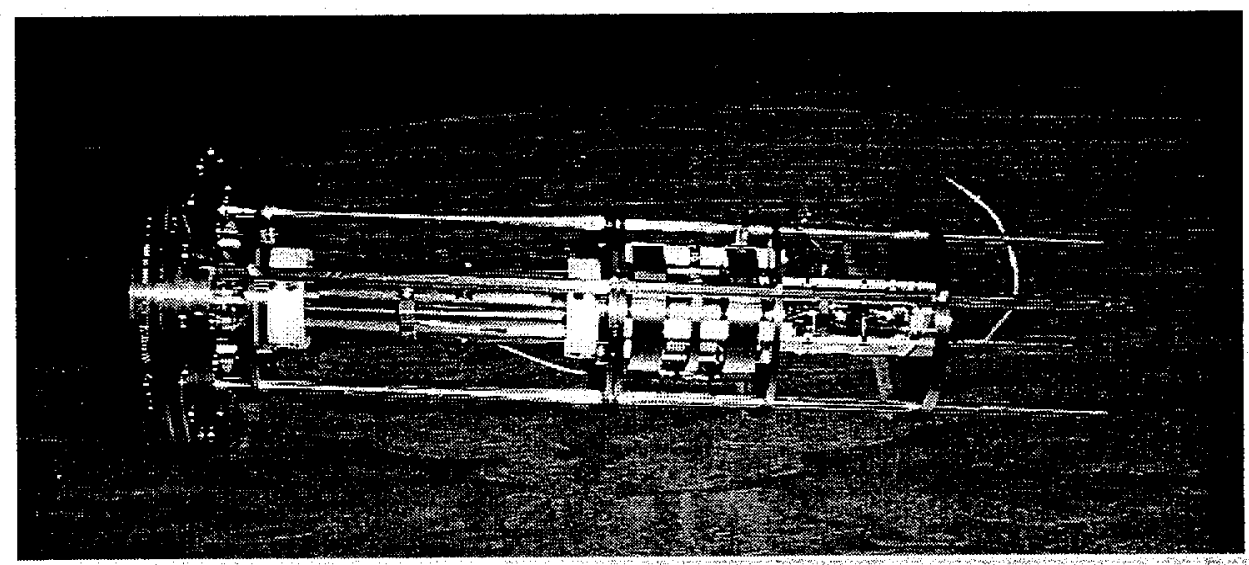

Figure 3: Photograph of microwave plasma source, quadrupole lens, and ion trap mass spectrometer

Not shown is the actual microwave cavity, which would be located just to the left of the vacuum 
flange shown in the figure.

Initial measurements of rare gases using a directly coupled MIP/ion trap mass spectrometer system (i.e. with no membrane introduction) were promising, [13] however sensitivity to environmentally important compounds was not as good as initially anticipated. Flow injection measurements using various analytes (ferrocene, triphenyltin chloride and aniline) showed distinct changes in the color of the plasma discharge, but little ion signal was observed in the mass spectrometer. Further, the MIP source introduced undesired analyte ion fragmentation, as can be seen for the example of aniline (Figure 4). The "reference" EI spectrum is shown in the top panel of the Figure, and the significant abundance of the $[\mathrm{M}-\mathrm{HCN}]^{+}$at $\mathrm{m} / \mathrm{z} 66$ ion is clear. Not only is this species prevalent in the argon MIP spectrum (bottom panel, Figure 4), but the $\left[\mathrm{M}-\mathrm{NH}_{2}\right]^{+}$ion at $\mathrm{m} / \mathrm{z} 77$ is also clearly visible. In contrast, the CE spectrum (center panel) is dominated by the molecular ion at $\mathrm{m} / \mathrm{z} 93$, which is important for both qualitative and quantitative MIMS analysis.
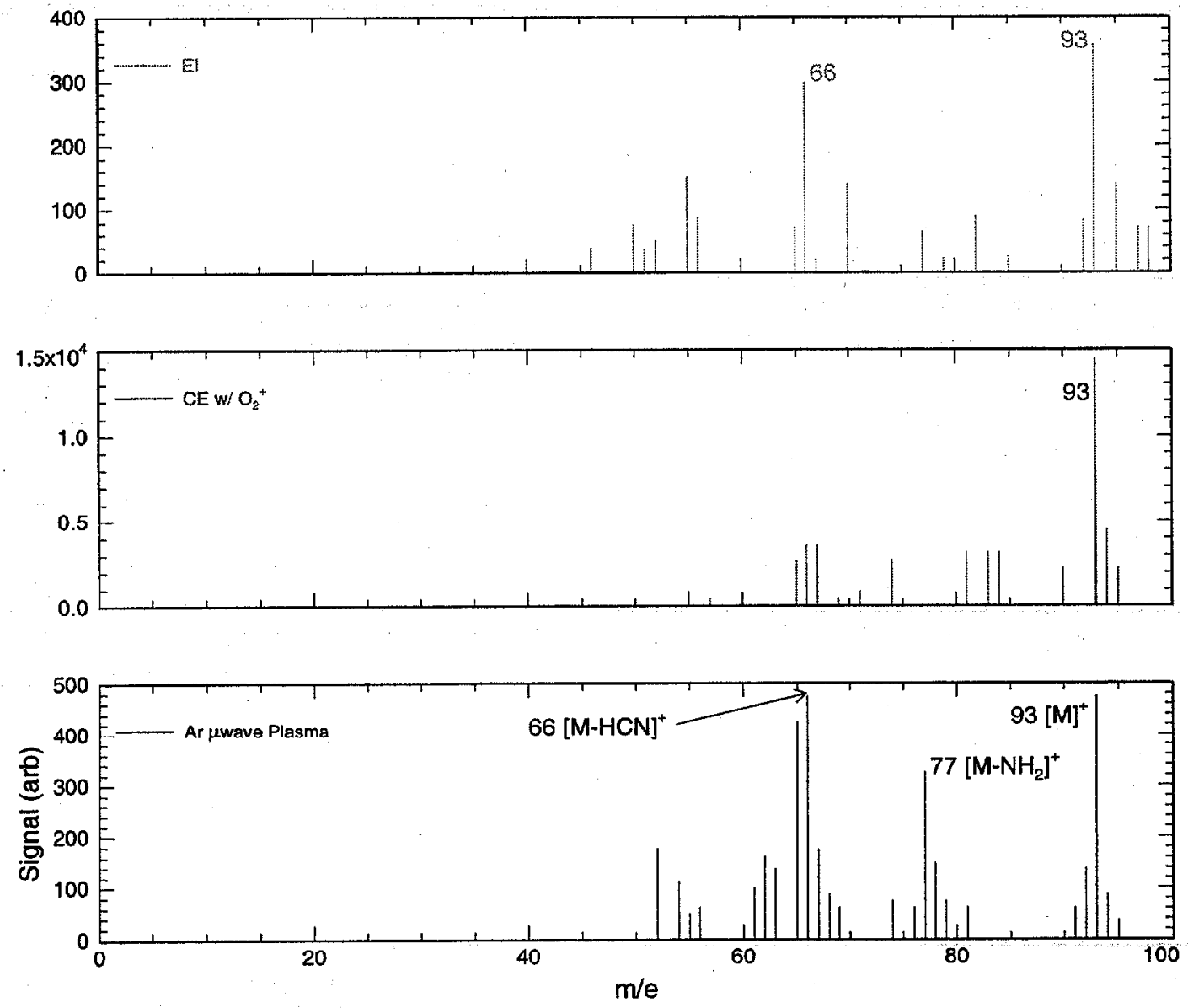

Figure 4: Comparison of fragmentation of Aniline $\left(\mathrm{C}_{6} \mathrm{H}_{5}-\mathrm{NH}_{2}\right.$ under different ionization conditions.

Detailed modeling of the instrument using the SIMION ion optics simulation package [14] and extensive experiments suggest that the lack of ion signal is due to poor ion transmission from the plasma discharge source to the ion trap mass spectrometer. A significant amount of further work 
would have been required to achieve the performance necessary for environmental characterization applications. However, we believe that charge exchange chemical ionization (as described above) provides outstanding detectability for our analytes of interest, and consequently we terminated the MIP/MIMS experiments.

\subsection{Simultaneous Detection}

One of the major goals of the project was to demonstrate the simultaneous detection of a broad spectrum of chemical contaminants with a single method/instrument. To date, we have used MIMS to detect 40 individual volatile and semi-volatile organic compounds (see Table 2) without preconcentration, from both air and water matrices. Other groups have demonstrated the ability to detect multiple compounds in a single chemical class with MIMS.[15, 16, 17, 18] The 40 volatile and semivolatile analytes range in boiling point from 21 to $283^{\circ} \mathrm{C}$ and include chlorinated and oxygenated solvents, chlorophenols, polycyclic aromatic hydrocarbons, and substituted benzenes. Essentially all of the compounds listed in Table 2 are identified as hazardous environmental contaminants by one or more regulatory agencies.

\begin{tabular}{|c|l|c|l|c|}
\hline & Compound Detected & b.p. $\left.{ }^{\circ} \mathrm{C}\right)$ & Vapor Pres. (torr) & MW \\
\hline \hline VOCs & Acetaldehyde & 21 & 750 & 44 \\
& Acetone & 56 & 180 & 58 \\
& Benzenc & 80 & 75 & 78 \\
& Carbon tetrachloride & 77 & 91.3 & 154 \\
& Chlorobenzene & 132 & 8.8 & 113 \\
& Hydroxy acetone & 131 & $10\left(39.7^{\circ} \mathrm{C}\right)$ & 74 \\
& 1-Hydroxy-2-butanone & 164 & $1\left(25.5^{\circ} \mathrm{C}\right)$ & 88 \\
& Isopropyl alcohol & 82 & 40 & 60 \\
& Methyl alcohol & 65 & 97.3 & 32 \\
& Methyl ethyl ketone & 80 & 100 & 72 \\
& Methyl isobutyl ketone & 117 & 16 & 100 \\
& 3-Pentanone & 102 & 27 & 86 \\
& Propanal & 49 & 300 & 58 \\
& Toluene & 111 & 22 & 92 \\
& Trichloroethane & 74 & 100 & 133 \\
& Trichloroethylene & 87 & 61 & 131 \\
\hline SVOCs & Acenaphthene & 283 & $10\left(131^{\circ} \mathrm{C}\right)$ & 154 \\
& Acetophenone & 203 & $1\left(15^{\circ} \mathrm{C}\right)$ & 120 \\
& Aniline & 184 & 0.6 & 93 \\
& 2-chlorophenol & 175 & $1\left(12.1^{\circ} \mathrm{C}\right)$ & 129 \\
& 3-chlorophenol & 214 & $1\left(44.2^{\circ} \mathrm{C}\right)$ & 129 \\
& Cyclohexanol & 161 & $1\left(21^{\circ} \mathrm{C}\right)$ & 100 \\
& 1,3-Dichlorobenzene & 173 & $1.9\left(25^{\circ} \mathrm{C}\right)$ & 151 \\
& 1,4-Dichlorobenzene & 174 & 0.6 & 147 \\
2,4-dichlorophenol & 210 & $1\left(53^{\circ} \mathrm{C}\right)$ & 163 \\
& 2-(diethylamino) ethanol & 162 & 1 & 117 \\
Diethyl malonate & 199 & $1\left(40^{\circ} \mathrm{C}\right)$ & 160 \\
& Diisopropyl aminoethanol & 187 & 0.1 & 145 \\
& Dimethyl methyl-phosphonate & 181 & $<0.1\left(10^{\circ} \mathrm{C}\right)$ & 124 \\
\hline \hline
\end{tabular}




\begin{tabular}{|l|l|c|l|c|}
\hline & Compound Detected & b.p. $\left({ }^{\circ} \mathrm{C}\right)$ & Vapor Pres. (torr) $^{2}$ & MW \\
\hline \hline \multirow{6}{*}{} & 2,4-Dimethylphenol & 210 & $1\left(51.8^{\circ} \mathrm{C}\right)$ & 125 \\
& Dimethyl sulfoxide & 189 & 0.4 & 78 \\
& 4-Hydroxy-4-methyl-2-pentanone & 164 & 1.1 & 116 \\
& Malathion & 157 & $0.7\left(157^{\circ} \mathrm{C}\right)$ & 330 \\
& Methyl salicylate & 223 & $1\left(54^{\circ} \mathrm{C}\right)$ & 152 \\
& 1-Methyl-naphthalene & 245 & $0.1 \mathrm{c}$ & 142 \\
& Naphthalene & 218 & 0.5 & 128 \\
& Nitrobenzene & 211 & 0.3 & 123 \\
& Phenol & 40 & $0.4\left(25^{\circ} \mathrm{C}\right)$ & 94 \\
& Pyruvic acid & $165^{4}$ & $1\left(21.4^{\circ} \mathrm{C}\right)$ & 88 \\
& 1,2,4-trichloro-benzene & 214 & $1\left(38.4{ }^{\circ} \mathrm{C}\right)$ & 181 \\
\hline Organo- & Ferrocene & 249 & $2.6\left(100^{\circ} \mathrm{C}\right)$ & 186 \\
metallics & Molybdenum hexacarbonyl & 156 & $0.1\left(25^{\circ} \mathrm{C}\right)$ & 266 \\
& Lead acetylacetonate & $\mathrm{NA}^{3}$ & $\mathrm{NA}^{3}$ & 405 \\
& Nickel acetylacetonate & $220^{4}$ & $11\left(220^{\circ} \mathrm{C}\right)$ & 257 \\
& Triphenyl tin chloride & 464 & $<1$ & 385 \\
\hline
\end{tabular}

Table 2: Compounds Detected by MIMS

We have also investigated the analysis of several organometallic compounds containing heavy metals by MIMS (see Table 2). When lead, tin, and nickel compounds were analyzed using MIMS, organic fragment ions were observed. However, neither the intact molecular ion nor the metal ion species were observed in the mass spectrum. Molecular ion species from both ferrocene and molybdenum hexacarbonyl, however, are readily detected in air following membrane exposure to headspace vapor from the solid.[19] The molecular ions, $\mathrm{m} / \mathrm{z} 186$ and 266, respectively, are the most abundant ions observed with charge exchange ionization. Less than $5 \%$ bare iron ( $\mathrm{Fe}^{+}$) or molybdenum $\left(\mathrm{Mo}^{+}\right)$, respectively, is observed under charge exchange conditions. Oxygen, as an ionized species $\left(\mathrm{O}_{2}^{+}\right)$, is one of the predominating species in the ion trap in our experiment and may be inhibiting the appearance of metal-containing fragment ions in certain cases. The high availability of $\mathrm{O}_{2}^{+}$for secondary reactions and the stability of the products from these secondary processes may make the detection of metal-containing ions problematic.

As a demoustration of the simultaneous detection capabilities of this technique, at least one compound from each class of compounds was selected for preparation as an aqueous mixture and as an air mixture. Figure 5 demonstrates the simultaneous detection of benzene (VOC), naphthalene (SVOC), and ferrocene (organometallic compound) in aqueous solution, and was obtained with charge exchange ionization using $\mathrm{H}_{3} \mathrm{O}^{+}$as the reagent ion from membrane-diffused water. The membrane was exposed to an aqueous sample containing the mixture of analytes for a period of 30 seconds (starting at 60 seconds and ending at 90 seconds). This was sufficient to detect $870 \mathrm{ppb}$ benzene, $150 \mathrm{ppb}$ naphthalene, and $180 \mathrm{ppb}$ ferrocene in water, as is illustrated by the response profiles (i.e. ion signals) obtained from each molecular ion: $\mathrm{m} / \mathrm{z} 78,128$, and 186 , respectively. The

\footnotetext{
${ }^{1}$ Boiling points, vapor pressure data, and molecular wcights arc from the respective Material Safety Data Sheets (MSDS).

${ }^{2}$ Vapor pressure data at sea level is reported at $20^{\circ} \mathrm{C}$ unless indicated otherwise.

${ }^{3} \mathrm{NA}$ : Not Available from MSDS.

${ }^{4}$ Decomposition temperature.
} 
benzene signal shows a sharp rise beginning at 125 seconds followed by a sharp decrease from 155 seconds to 180 seconds. The response time lag of more volatile compounds like benzene is expected to be about 60 seconds, and represents the minimum time necessary for highly volatile analytes to travel through the transfer lines from the sample container to the membrane assembly, and finally to the ion trap mass spectrometer. Naphthalene $(\mathrm{x} 5)$ and ferrocene $(\mathrm{x} 20)$, on the other hand, show more gradual increases and decreases in ion signals, indicative of the longer diffusion times required for the less volatile compounds (than benzene) to pass through the membrane into the sample transport gas (i.e. helium). Permeation of the analyte through the membrane is governed directly by the diffusivity and solubility constants with diffusion being the rate-limiting step for species with similar solubility.[1]

\section{MIMS of VOC/SVOC/Organometal Sample in Water}

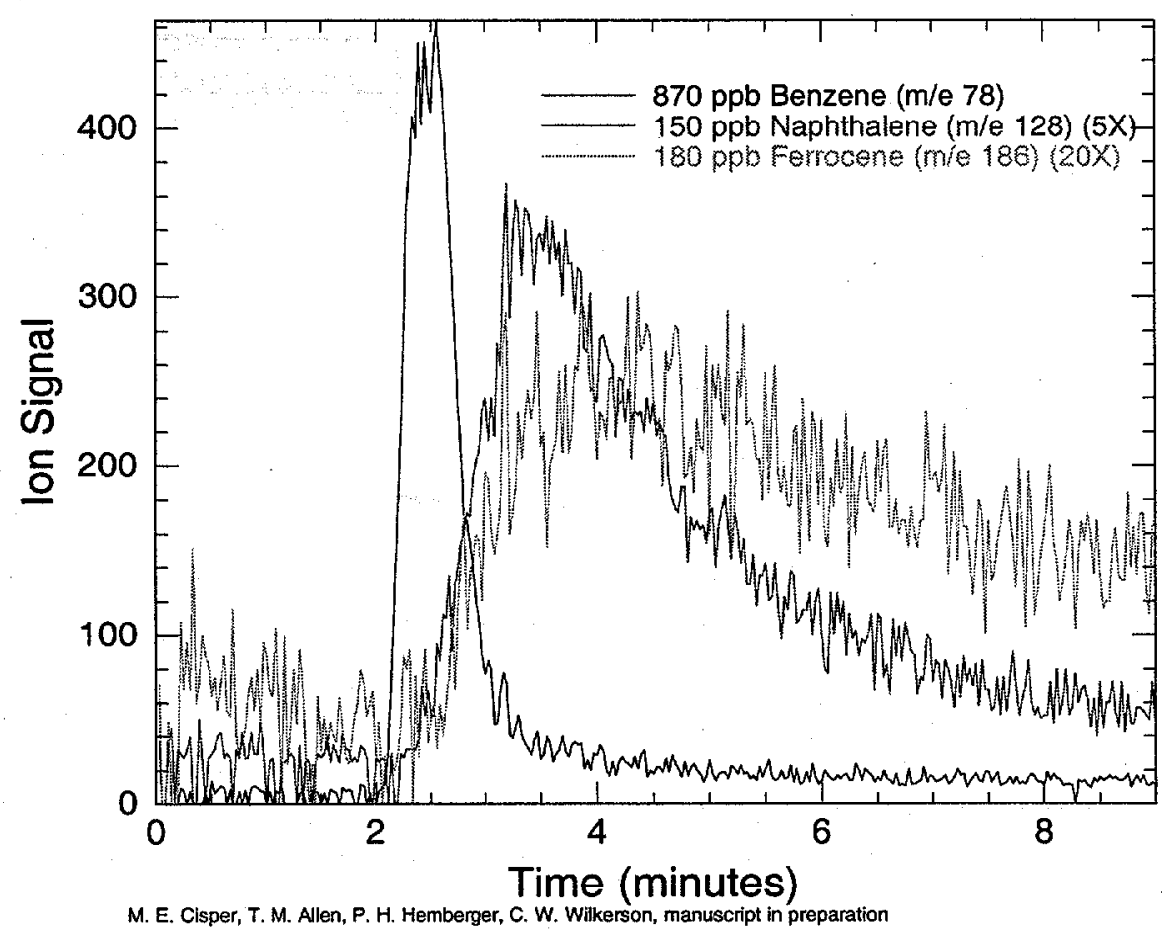

Figure 5: Demonstration of Simultaneous Detection of VOCs, SVOCs, and Organometallic Compounds in Water

The same membrane assembly used for the aqueous mixture (Figure 5) is also suitable for the detection of a mixture of two VOCs, one SVOC, and one organometallic compound in air (Figure 6). Figure 6 illustrates the simultaneous detection of $660 \mathrm{ppb}$ methyl ethyl ketone (VOC), $7 \mathrm{ppb}$ toluene (VOC), $316 \mathrm{ppb}$ 1-methylnaphthalene (SVOC), and $700 \mathrm{ppb}$ ferrocene (organometallic) in air, and was obtained with charge exchange ionization using $\mathrm{O} 2^{+\cdot}$ as the reagent ion from membrane-diffused air. A sampling period of 2 minutes was begun at at 60 seconds and ended at 180 seconds. The response profile of the more volatile compounds, methyl ethyl ketone (MEK) and toluene, matches the sampling time period of 2 minutes. A time lag of about 30 seconds, corresponding to the time necessary for highly volatile analytes to travel through the transfer lines 
from the Dynacalibrator, the membrane assembly, and finally the ion trap mass spectrometer, is observed for MEK and toluene. The response profiles of MEK ( $x$ 10) and toluene ( $x$ 25) are depicted by very sharp increases in the molecular ion signals $(\mathrm{m} / \mathrm{z} 72$ and 92 , respectively) followed by sharp decreases. Slower compound diffusivity is observed for the less volatile compounds of 1-methylnaphthalene (MNAP) and ferrocene. The response time lag of the membrane to MNAP is 70 seconds after sample release from the Dynacalibrator. The total response profile of MNAP $(\mathrm{m} / \mathrm{z}$ 142), illustrated in Figurc 6 by the gradual decrease in the molecular ion signal, extends beyond the 2 minute sample exposure time by greater than one minute. The response profile of ferrocene (x 60, m/z 186) shows a weaker response than MEK, toluene, and MNAP. The ferrocene response time lag is approximately 60 seconds.

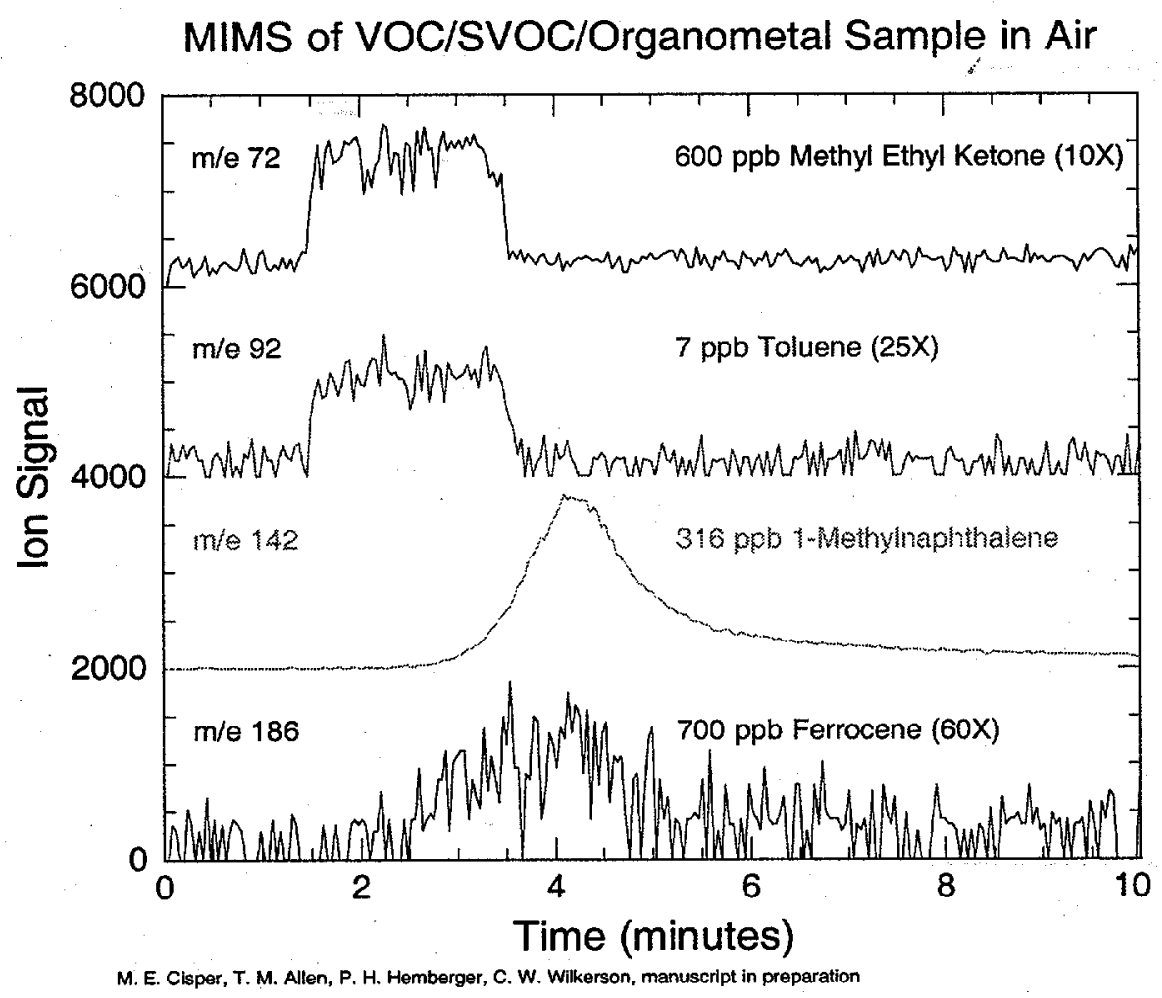

Figure 6: Demonstration of Simultaneous Detection of VOCs, SVOCs, and Organometallic Compounds in Air

\subsection{Tailored Membranes}

During FY00 we conducted experiments aimed at tuning the selectivity of pervaporation membranes in order to optimize the measurement of specific classes of hazardous chemicals. Silicone (PDMS) membranes are well-suited for real-time analysis of non-polar and moderately polar volatile organic compounds. However, analyses of more polar and less volatile analytes are often more difficult with a silicone membrane, because of decreased solubility and diffusivity issues. In collaboration with R. B. Timmons (Dept. of Chemistry, University of Texas at Arlington), we have developed new membranes with tunable properties that will allow us to largely avoid such problems. Both the 
nature of the monomer (e.g. dimethylsiloxane, allyl alcohol, methylamine, etc.) and the conditions under which it is polymerized (pressure, plasma duty cycle, final film thickness) can be controlled to achieve desired membrane performance.

The ability to control the hydrophobicity of the membrane coating, and hence the selectivity of the membrane, through the use of variable plasma polymerization conditions is in our opinion the most important result of this research. Plasma polymerization conditions may be controlled by adjusting a variety of experimental variables, such as plasma power, duty cycle, and overall reaction time, as well as the identity of the precursor monomer. Even by adjusting only one of these parameters, plasma duty cycle, dramatic changes in the coating may be achieved.[20] For example, when using allyl alcohol (2-propene-1-ol) as the monomer, the hydrophobicity of the coating can be controlled by simply varying the ratio of plasma "off" time to "on" time (duty cycle), as demonstrated in the infrared spectrum of several allyl alcohol coatings, shown in figure 7. When the duty cycle is low (for example, in the case where the plasma is on for $1 \mathrm{msec}$ but off for 10-30 msec) the resultant polymer retains a large fraction of its hydroxyl functionality (as evidenced by the retention of the broad $\mathrm{O}-\mathrm{H}$ stretch peak in the IR spectrum at $\approx 3400 \mathrm{~cm}^{-1}$, and the membrane is much more hydrophilic. On the other hand, the 1:2 msec ratio results in a film that does not maintain its $-\mathrm{OH}$ groups, resulting in a more hydrophobic coating.

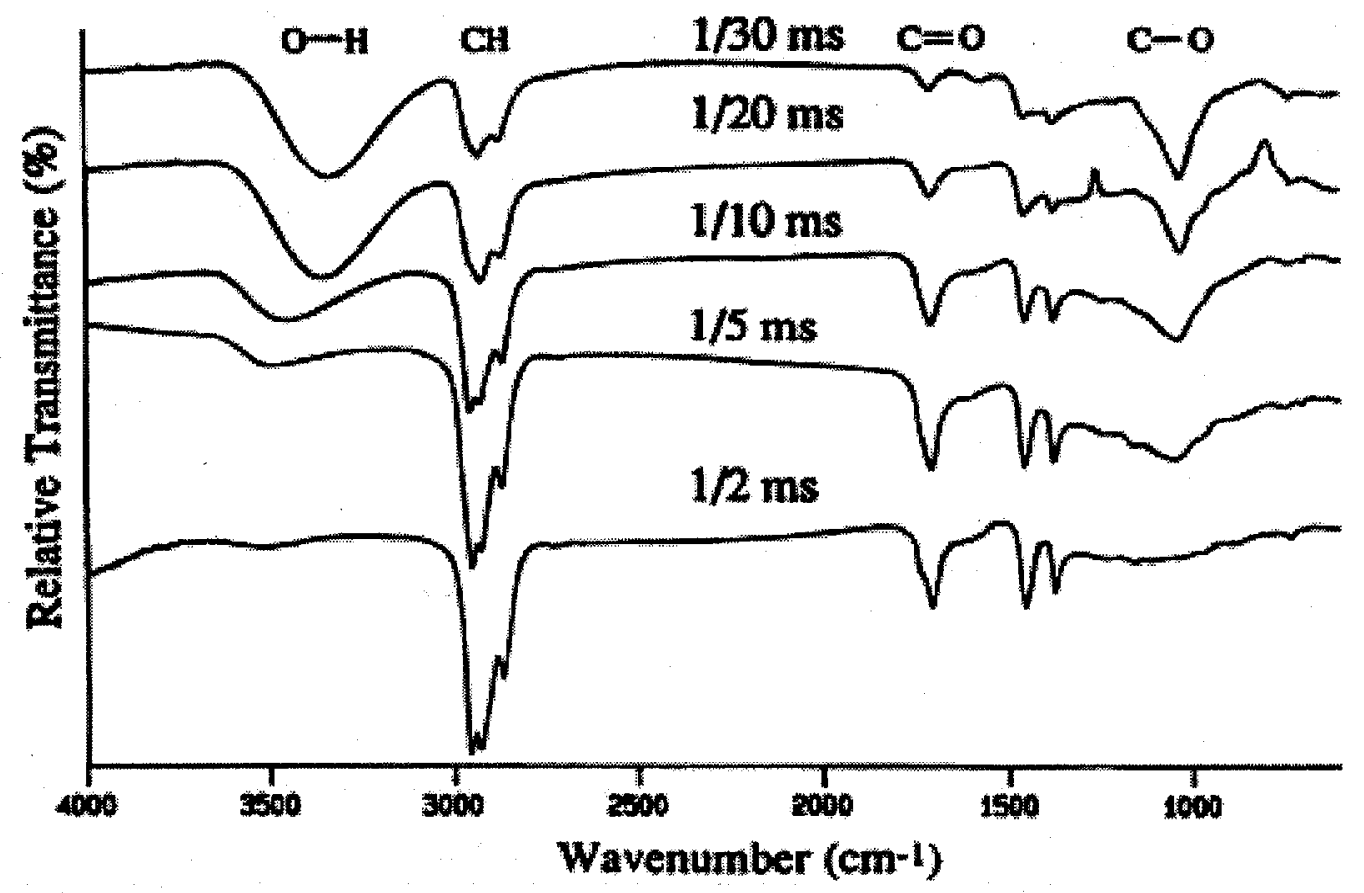

Figure 7: Infrared Spectra of poly(allyl alcohol) Membranes [20]

Preliminary data (vide infra) show that, compared to a PDMS membrane, a particular plasmadeposited allyl alcohol membrane enhances the detection of polar organic compounds while simultaneously suppressing the detection of non-polar species. By simply changing the plasma duty cycle during polymerization, this same monomer produces a membrane with completely reversed MIMS properties (i.e. suppression of polar, and transmission of non-polar, analytes). We feel that exploitation of this new paradigm in MIMS will allow the development of a truly broad-spectrum technique. 
We studied several analytes during the tailored membrane developmental experiments, but here we focus on methanol as a prototypical polar species. MIMS measurements were carried out using two different membrane coatings. Commercially available polydimethylsiloxane fibers provide a relatively non-polar interface, whereas the allyl alcohol custom-coated fibers afford a more hydrophilic environment. Bolh membranes are synthesized via plasma polymerization of the monomer onto the outer surface of a microporous polypropylene hollow support fiber $(209 \mu \mathrm{m}$ i.d. x $263 \mu \mathrm{m}$ o.d.).[20, 21] Pulsed plasma polymerization of allyl alcohol deposits a high hydroxyl surface content onto the support fiber, and the extent of hydrophilicity of the allyl alcohol coating can be controlled by the plasma "on" and "off" times (i.e. the duty cycle).[20] Polymerization of a gas plasma containing a fully alkylated (e.g. hexamethyl) disiloxane and oxygen produces a high methyl content on the support fiber.[21]

The utility of an analytical method such as membrane introduction mass spectrometry depends upon, among other things, its linear dynamic range and reproducibility. Figure 8 illustrates two linear calibration curves for methanol $(\mathrm{m} / \mathrm{z} 33)$ in air using charge exchange ionization. The lower plot in Figure 9 displays methanol from 14 to $599 \mathrm{ppm}$ in air $\left(27^{\circ} \mathrm{C}\right)$ taken with the PDMS membrane over a two day period, and illustrates measurement reproducibility and instrument stability. The upper plot in Figure 2 shows methanol from 3.3 to $701 \mathrm{ppm}$ in air $\left(27^{\circ} \mathrm{C}\right)$ obtained with the "1:15" AA membrane (duty cycle of $1 \mathrm{msec}$ "on" and $15 \mathrm{msec}$ "off"). Calibration points in Figure 9 were obtained by exposing alternating blank air and methanol air samples to the membrane. Each calibration point represents the average peak area measurement obtained by sampling each concentration 3-8 times for sampling periods of 30 seconds. Error bars indicate one standard deviation from the average area.

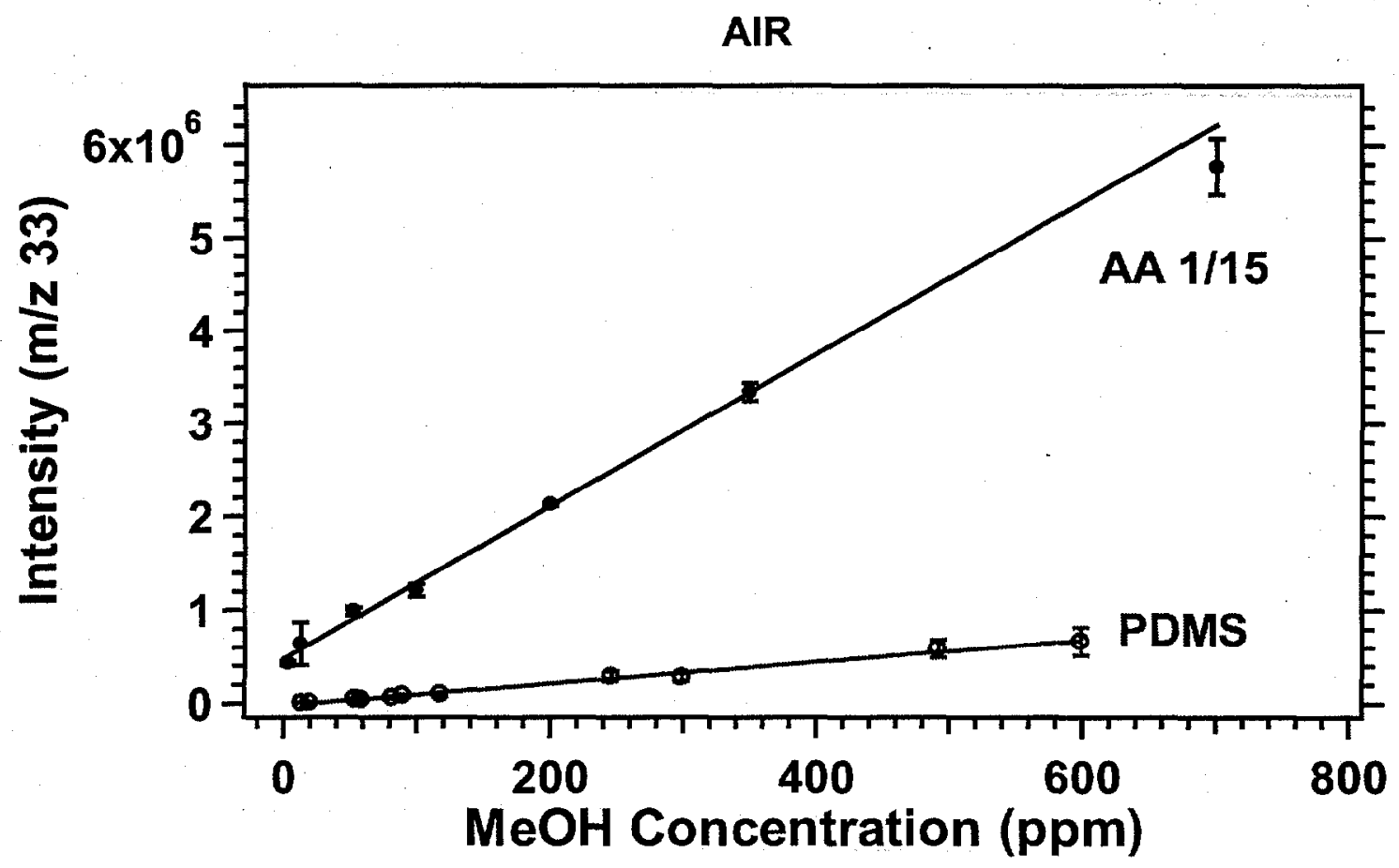

Figure 8: Comparison of MIMS response to methanol in air as a function of concentration for PDMS (red, lower trace) and "1:15". AA (blue, upper trace) membranes 
Utilization of the AA membrane, as compared to PDMS, significantly improved the sensitivity of MIMS in the detection of methanol in air. The AA membrane demonstrates enhanced sensitivity over the PDMS membrane by a factor of seven (based on the slopes obtained from the linear calibration curves). The lower limit for detecting methanol $\left([\mathrm{M}+\mathrm{H}]^{+}\right)$in air was reduced by 4.2 times from $14 \mathrm{ppm}$ for the PDMS membrane to $3.3 \mathrm{ppm}$ for the AA membrane. Detection limits are defined at a signal to noise ratio of three. Both lower limits of $14 \mathrm{ppm}$ (PDMS) and $3.3 \mathrm{ppm}$ (AA) are more than an order of magnitude below the current threshold exposure limit of $200 \mathrm{ppm}$ (also reported as $262 \mu \mathrm{g} / \mathrm{m}^{3}$ ) in air.[22] Both PDMS and AA membranes demonstrate a linear dynamic range for methanol detection in air that extends beyond 2 orders of magnitude. The maximum concentration determined in our measurements is limited by the Dynacalibrator; the ultimate upper limit to the linear dynamic range may be higher based on our analysis of methanol in water (discussed below).

Figure 9 shows two linear calibration curves for methanol $(\mathrm{m} / \mathrm{z} 33)$ in water using charge exchange ionization. The same PDMS and AA membranes were used for both air and water experiments. The bottom plot in Figure 9 shows methanol from 5 to $1000 \mathrm{ppm}$ in water taken with the PDMS membrane at room temperature $\left(24^{\circ} \mathrm{C}\right)$, and the top plot depicts methanol from 2 to $100 \mathrm{ppm}$ in water obtained with the AA membrane at room temperature $\left(26^{\circ} \mathrm{C}\right)$. Similar to the data shown in Figure 8 for methanol in air, the calibration points in Figure 9 were obtained by exposing alternating blank water and aqueous methanol solutions to the membrane. Again, each point represents the average peak area measurement obtained by sampling each concentration 3-5 times for sampling periods of 30 seconds, and error bars indicate one standard deviation from the avcrage area.

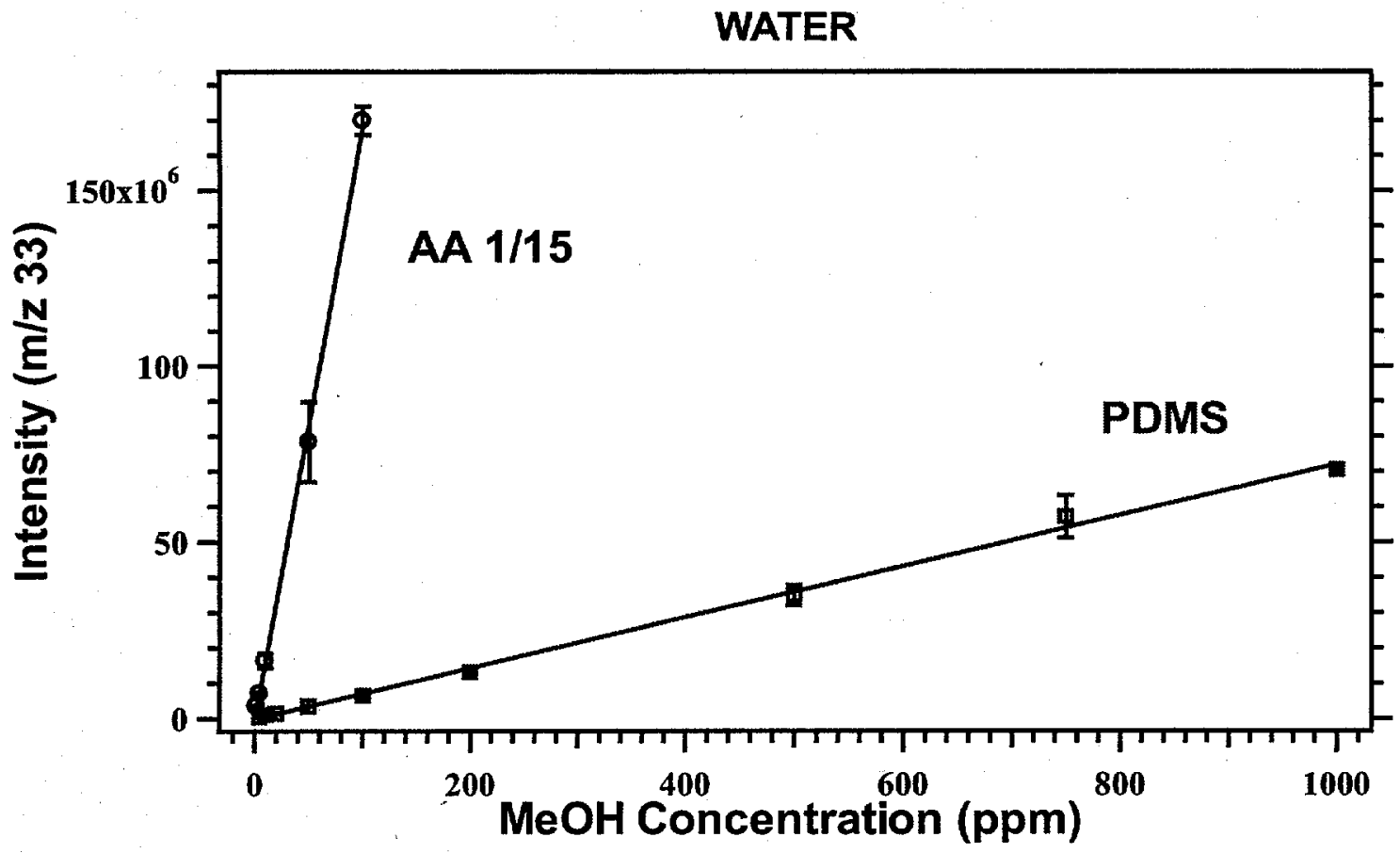

Figure 9: Comparison of MIMS response to methanol in water as a function of concentration for PDMS (red, lower trace) and "1:15" AA (blue, upper trace) membranes 
Utilization of the AA membrane, as compared to PDMS, had a very dramatic affect on the sensitivity of MIMS in the detection of methanol in water. As can be seen in Figure 9, the linear dynamic range of the AA membrane is much lower, nearly 2 orders of magnitude, than that of the PDMS membrane, which extends well beyond 3 orders of magnitude. In the AA membrane experiments, quantification of $250 \mathrm{ppm}$ (or greater) methanol $\left(\mathrm{m} / \mathrm{z} 33,[\mathrm{M}+\mathrm{H}]^{+}\right)$in water was thwarted by space charging, which typically occurs with $\approx 10^{6}$ ions in the ion trap mass spectrometer.[23] Although the linear dynamic range is lower, the AA membrane demonstrates significantly enhanced (23.4 times over the PDMS membrane) sensitivity to low (2-100 ppm) concentrations of methanol in water. The lower limit of detection of methanol in water was reduced by 2.5 times from $5 \mathrm{ppm}$ for the PDMS membrane to $2 \mathrm{ppm}$ for the AA membrane. A background peak at $\mathrm{m} / \mathrm{z} 33$ for blank water samples, which was not observed for blank air samples, most likely results from protonated oxygen $\left(\left[\mathrm{HO}_{2}\right]^{+}\right)$. Protonated methanol was observed in each of the 30 second sampling periods of $5 \mathrm{ppm}$ (PDMS) and for $2 \mathrm{ppm}$ (AA) methanol in water, but was not observed in any sampling periods for $1 \mathrm{ppm}$ (PDMS) and $500 \mathrm{ppb}(\mathrm{AA})$ methanol in water.

In each set of experiments (air or water samples), the same PDMS and AA membranes were used for detecting methanol. For both membranes, the sensitivity of MIMS was greater for methanol in water than for methanol in air: 207 times greater for AA and 62 times greater for PDMS. Even though the AA and PDMS membranes act as suitable barriers between the sample and the vacuum of the mass spectrometer, neither membrane will completely exclude water (or air) from diffusing through the membrane and entering the mass spectrometer. More methanol, which is completely miscible in water, is expected to diffuse through the membranes with water samples than with air samples. Additionally, more water and methanol are expected to diffuse through the AA membrane (high -OH content) since it is a more hydrophilic membrane surface than PDMS (high $-\mathrm{CH}_{3}$ content). For example, concentrations greater than $250 \mathrm{ppm}$ methanol in water with the AA membrane resulted in space charging at $\mathrm{m} / \mathrm{z} 33$, the quantification ion. Space charging was not observed for either membrane with any of the air samples of methanol.

In addition to improving sensitivity, tailored membranes can be used to provide selectivity to MIMS measurements. This is demonstrated in Figure 10. In this experiment, five analytes (methanol, acetone, methyl ethyl ketone, benzene, and naphthalene) were analyzed independently (as opposed to simultaneously) using three different membranes: the "standard" commercial PDMS coating, the "1:15" AA coating (used above for the methanol sensitivity studies), and a "1:2" AA coating, which is expected to be more hydrophobic.[20]

The performance of the PDMS membrane (red bars, far left bar in each set) is fairly consistent across the analyte spectrum. This is expected from our previous experience. The "1:15" AA membrane (green bars, center bar in each set) shows a significant suppression of the response from the less polar analytes (benzene, naphthalene), while enhancing the response of the more polar species (methanol, acetone, and MEK). By simply changing the duty cycle of the polymerization process, the "1:2" AA coating (blue bars, far right bar in each set) generates the opposite effect: polar species are suppressed, while the less polar polycyclic aromatic hydrocarbons (PAHs) have an improved signal level. This is an exciting result, that suggests an interesting possibility for site characterization.

Employing multiple membrane assemblies, each with a tailored coating that would preferentially pervaporate certain chemical species (or classes of analytes), the screening tool could be quickly customized to the expected composition of the waste site. For example, if PAHs are thought to be predominant, a non-polar-specific membrane would be used; or if chelated metals are suspected, a tailored coating for the chelator would be indicated. Because of the small size and simple design of our membrane assemblies, many selective units could be pre-built and included in the deployed unit. The technician would simply gang together as many assemblies as believed necessary, along 
with a broad spectrum (PDMS, for example) membrane, and perform the on-site analysis.

We have performed some preliminary experiments where dual membrane assemblies are connected in either a parallel or serial fashion (Figures 11 and 12). Our brief experience (these measurements were initiated in the final quarter of FY00) suggests that the parallel arrangement is preferred, because this retains the speed of the overall analysis.

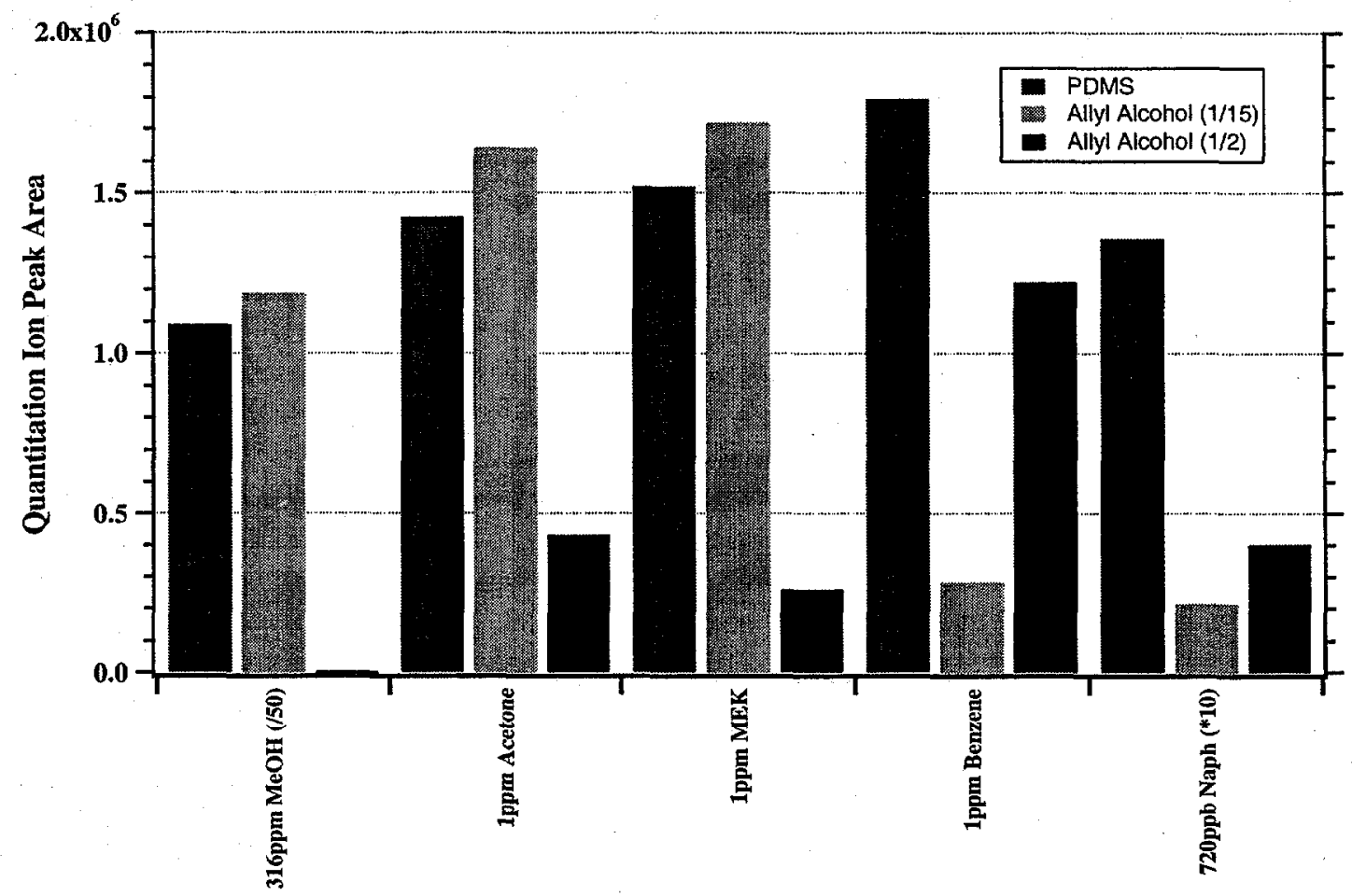

Figure 10: Comparison of membrane response as a function of coating chemistry for a small selection of analytes.

While we have not made parallel measurements using different membrane chemistries, an example using a dual PDMS system is shown in Figure 13. In this experiment, two injection pulses of ethanol (10 ppm), acetone (10 ppb), toluene (10 ppb), and benzene (10 ppb) are made into a sampling system as shown in Figure 11. One can see that for each injection pulse, there are two response signals in the ion trap: the first due to the sample plug moving through the first membrane assembly, and the second resulting from the later passage through the second membrane. The time delay is proportional to the length of tubing connecting the two assemblies. From these data we could also estimate the efficiency of analyte removal from the first membrane if both of the assemblies had been calibrated. 


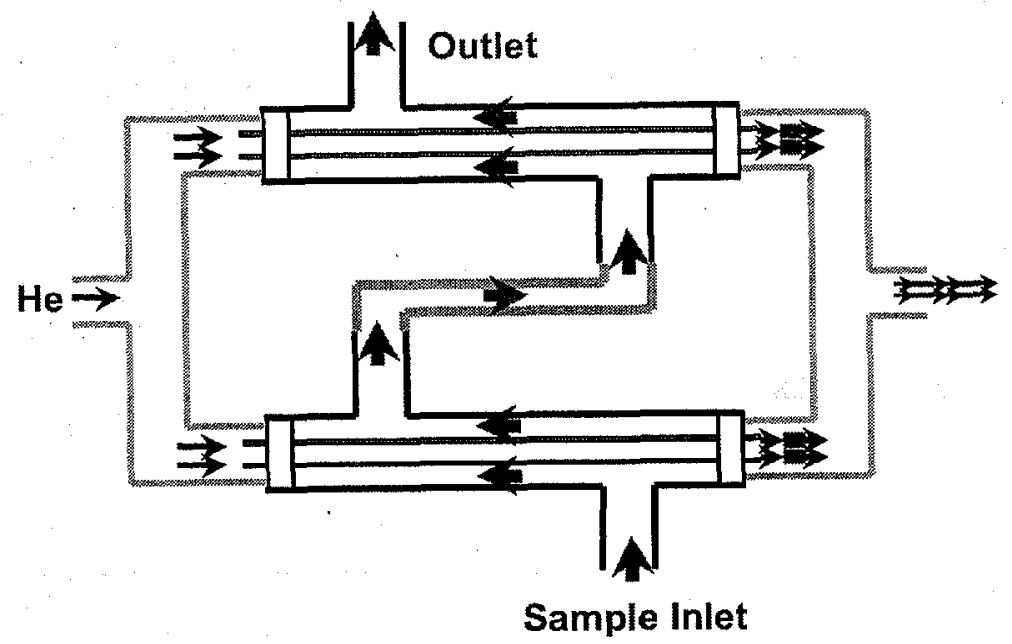

Figure 11: Connection_of tailored membrane assemblies in parallel.

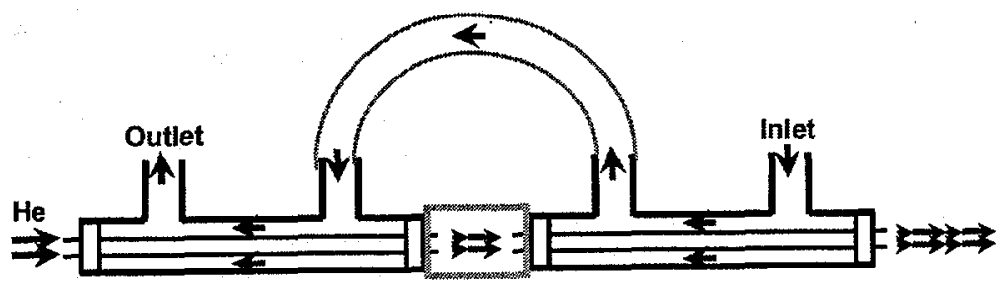

Figure 12: Conncction of tailored membrane assemblies in serial.

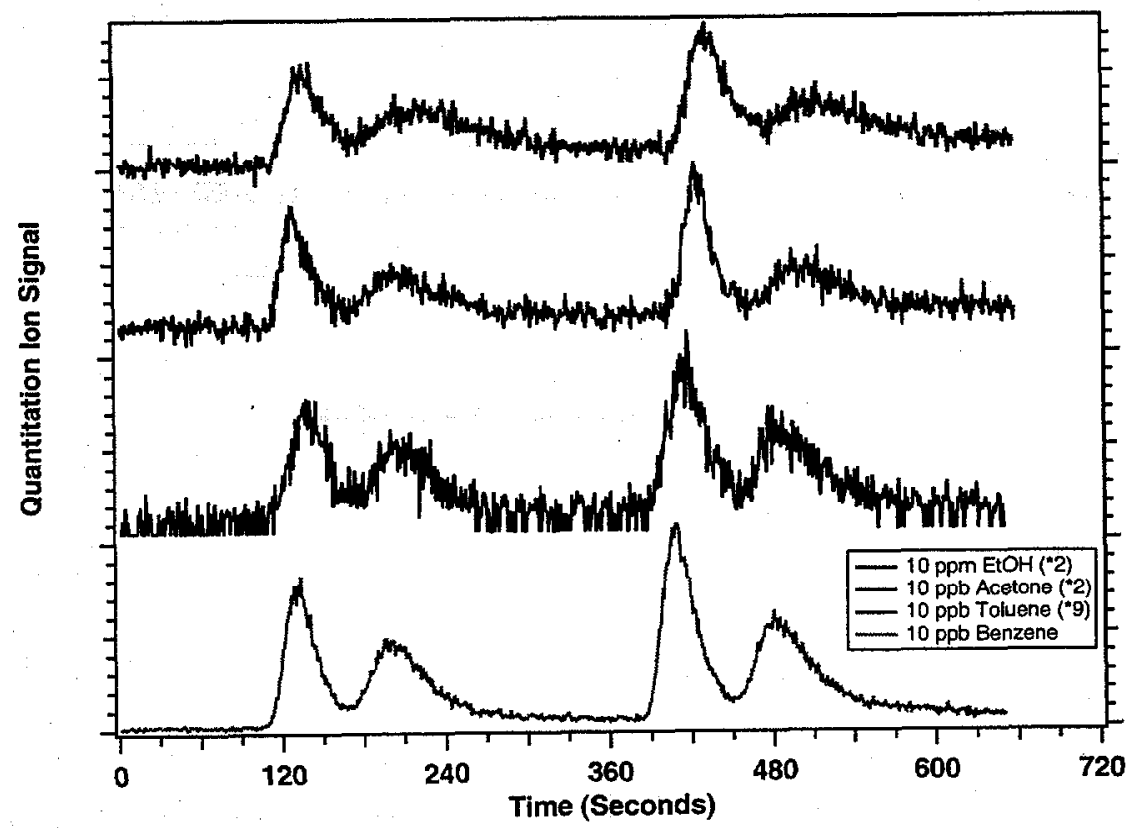

Figure 13: Test of multiple-membrane geometry with dual PDMS membranes 


\section{Relevance, Impact and Technology Transfer}

How does this new scientific knowledge focus on critical DOE environmental management problems? Characterization of Mixed Wastes is an important problem for the DOE. Timely site screening can significantly impact the resources needed to expedite cleanup planning and execution.

How will the new scientific knowledge that is generated by this project improve technologies and cleanup approaches to significantly reduce future costs, schedules, and risks and meet DOE compliance requirements? As discussed above, by facilitating rapid sample screening, site characterization may be speeded, and the number of samples requiring extensive laboratory-based analyses reduced. Technology developed using the results of our research will provide the tools to minimize the required time and resources to obtain an accurate picture of the chemical hazards associated with a hazardous or mixed waste site.

To what extent does the new scientific knowledge bridge the gap between broad fundamental research that has wide-ranging applications and the timeliness to meet needs-driven applied technology development? Because we have demonstrated the ability to tune the selectivity of the membrane, and the feasibility of applying this technology to the simultaneous detection of a broad range of chemical species, we believe that it is evident that this work has spanned the spectrum from very basic research to needs-driven application.

What is the project's impact on individuals, laboratories, departments, and institutions? Will results be used? If so, how will they be used, by whom, and when? It remains to be seen if the approach that we have pursued will be implemented at any level in the DOE. We believe that this was a successful project, with a good prognosis for real world application. The most likely scenario for deployment of this technology would be as a rapid screening tool for site characterization, as a "go/no-go" arbiter for selecting samples for laboratory measurements.

Are larger scale trials warranted? What difference has the project made? Now that the project is complete, what new capacity, equipment or expertise has been developed? For liquid and air monitoring, we believe that this technology is sufficiently mature that no further testing is needed (except for radionuclide issues (vide infra). We have produced a fieldable prototype instrument, albeit one that still requires a highly trained operator. Given the interest, desire, and support, it would be relatively straightforward to produce multiple copies of the instrument with control and analysis software that could be operated by competent chemical technicians. Soil matrices need to investigated at the laboratory. scale before this sensor could be routinely applied to solid waste streams.

How have the scientific capabilities of collaborating scientists been improved? Our interactions with Professor Timmons have been mutually beneficial: we have learned how to apply polymer materials science to environmental problems, and I believe that the UT-Arlington group has found another area of application for their very clever plasma polymerization techniques. The collaboration with the University of North Dakota provided summer experiences in environmental analytical chemistry to one undergraduate student and one graduate student.

How has this research advanced our understanding in the area? We have made several basic contributions to the MIMS literature, and our work in FY00 involving chemically tailored membranes clearly shows that we can use controlled techniques to enhance analyte selectivity. While this is a somewhat obvious path to have followed, it has not been previously explored, and is potentially a significant advance in the area.

What additional scientific or other hurdles must be overcome before the results of this project can be successfully applied to DOE Environmental Management problems? One issue that this work did not address was confirmation of radionuclide exclusion from the 
instrument. Clearly it is important to insure that the membrane assembly, which is inexpensive and small, is the only part of the device that becomes contaminated during operation. These measurcments were not within the scope of the EMSP project, but need to be performed before this technology could be applied to DOE mixed waste sites.

Have any other government agencies or private enterprises expressed interest in the project? Please provide contact information. We have not had any formal solicitations from other agencies or companies, but we have had a number of positive comments from colleagues at professional meetings, and interest from a few visitors to the project poster at the EMSP Workshop last spring in Atlanta.

\section{Project Productivity}

This project accomplished its defined goal: to extend MIMS to semivolatile and metal-containing contaminants. A list of the compounds that we have successfully detected with this technique is presented in Table 2.

\section{Publications}

This project has resulted in the following publications:

1. "Simultaneous Detection of VOCs, SVOCs, and Organometallic Compounds in Air and Water by Membrane Introduction Mass Spectrometry." T. M. Allen, M. E. Cisper, P. H. Hemberger, and C. W. Wilkerson. Invited Paper in preparation for the International Journal of Mass Spectrometry.

2. "Real-time Analysis of Methanol in Air and Water by Membrane Introduction Mass Spectrometry." T. M. Allen, T. M. Falconer, M. E. Cisper, A. J. Borgerding, and C. W. Wilkerson. Submitted to Analytical Chemistry.

3. "Environmental Monitoring" in Membrane Introduction Mass Spectrometry, M. E. Cisper, T. M. Allen, T. Kotiaho, R. Ketola; Editors: R. Graham Cooks and Tapio Kotiaho, J. Wiley \& Sons, Inc., Invited book chapter. In Preparation.

4. "Membrane Introduction Mass Spectrometry: Trends and Applications." R. C. Johnson, R. G. Cooks, T. M. Allen, M. E. Cisper, and P. H. Hemberger. Mass Spectrometry Reviews, $2000,19,1-37$.

5. "Real-Timc Broad Spectrum Characterization of Waste by Membrane Introduction Mass Spectrometry: Preliminary Results." T. M. Allen, M. E. Cisper, C. W. Wilkerson, and P. H. Hemberger. Waste Management Conference Proceedings, Abstract \#820, Tucson, AZ, 1999.

\section{Interactions}

We have presented the results of our work at a number of national conferences:

- ACS National Meeting, August 1999, New Orleans, LA.

- 46th Conference on Mass Spectrometry and Allied Topics, June 1999, Dallas, TX.

- 48th Conference on Mass Spectrometry and Allied Topics, June 2000, Palm Springs, CA. 
- Pittcon, March 1999, New Orleans, LA.

- WM99, Tucson, AZ.

There have also been invited seminars on our work at the following universities:

- Arizona State University

- University of North Dakota

- University of 'Texas - Arlington

\section{Transitions}

Membrane introduction mass spectrometry is being pursued by a number of research groups around the world, and our work has added to the body of knowledge for the field. This technique is used in process and environmental monitoring, and by academic, industrial, and government scientists. We are not aware of any specific uses of our EMSP results, but our work has been well-received at all of the scientific meetings where it has been presented. Citation of our research publications will ultimately indicate the significance of the project in the MIMS research community.

\section{Patents}

No patents were generated from the work executed in this project.

\section{Future Work}

The further development of chemically tailored membranes may lead to significant improvements in this methodology, particularly for environmental applications. We submitted a proposal to pursue such studies, but it has not yet been funded. Due to other commitments on the part of the PI, and lack of support to retain the needed personnel, it is unlikely that this project will continue.

\section{References}

[1] R. C. Johnson, R. G. Cooks, T. M. Allen, M. E. Cisper, and P. H. Hemberger, Mass Spectrom. Rev., 2000, 19, 1-37.

[2] N. Srinivasan, R.C. Johnson, N. Kasthurikrishnan, P. Wong, and R. G. Cooks, Anal. Chim. Acta, 1997, 350, 257-271.

[3] J. N. Louris, R. G. Cooks, J. E. P. Syka, P. E. Kelley, G. C. Stafford, Jr., and J. F. J. Todd, Anal. Chem., 1987, 59, 1677-1685.

[4] R.C. Johnson, R.G. Cooks, T.M. Allen, M.E. Cisper, and P. H. Hemberger, Mass Spectrom. Rev., 2000, 19, 1-37.

[5] P. S. H. Wong and R. G. Cooks, Anal. Chim. Acta, 1995, 310, 387-398.

[6] M. E. Cisper, A. W. Garrett, D.Cameron, and P. H. Hemberger, Anal. Chem., 1996, 68, $2097-2101$. 
[7] M. E. Cisper, A. W. Garrett, D. Cameron, and P. H. Hemberger, Anal. Chem., 1996, 68(13), 2097-2101.

[8] J. Poths and E. P. Chamberlin, IJMSIP, 1995, 146/147, 47-54.

[9] J. S. Ho W. L. Budde L. E. Slivon, M. R. Bauer, Anal. Chem., 1991, 63(13), 1335-1340.

[10] P. H. Dawson, Quadrupole Mass Spectrometry; Elsevier Scientific Publishing Company, Amsterdam, 1976.

[11] C. D. Cleven, R. G. Cooks, A. W. Garrett, N. S. Nogar, and P. H. Hemberger, J. Phys. Chem., 1996, $100(1), 40-46$.

[12] A. W. Garrett, M. E. Cisper, N. S. Nogar, and P. H. Hemberger, $R C M S$, 1994, 8(2), 174-178.

[13] M. E. Cisper, A. W. Garrett, Y. X. Duan, J. A. Olivares, and P. H. Hemberger, IJMSIP, 1998, 178, 121-128.

[14] D. A. Dahl; SIMION 3D Version 6.0; In 43rd ASMS Conference on Mass Spectrometry and Allied Topics, page 717, Atlanta, GA, 1995.

[15] K. Bennett and K. Cook; Zeolite membrane-based membrane inlet mass spectrometry; In 46th Conference on Mass Spectrometry and Allied Topics, 1998.

[16] C. Xu, J. Patrick, and R. G. Cooks, Anal. Chem., 1995, 67, 724-728.

[17] R. G. Cooks and T. Kotiaho; Membrane introduction mass spectrometry in environmental analysis; In ACS Symp. Ser., pages 126-154, 1992.

[18] C. S. Creaser and J. W. Stygall, Anal. Proc., 1995, 32, 286.

[19] M. E. Cisper and P. H. Hemberger, Rapid Commun. Mass Spectrom., 1997, 11, 1454-1456.

[20] C. L. Rinsch, X. L. Chen, V. Panchalingam, R. C. Eberhart, J. H. Wang, and R. B. Timmons, Langmuir, 1996, 12(12), 2995-3002.

[21] H. Nomura; Gas plasma polymerized permselective membrane, U. S. Patent 5,459,736, 1995.

[22] MSDS, Methyl Alcohol; MDL Information Systems, Inc., 1998.

[23] R. E. March and J. F. J. Todd, Practical Aspects of Ion Trap Mass Spectrometry; CRC Press, Boca Raton FL, 1995. 